\title{
Super-convergence of Discontinuous Galerkin Method Applied to the Navier-Stokes Equations
}

\author{
Harold L. Atkins* \\ NASA Langley Research Center, Hampton, VA 23681-2199
}

\begin{abstract}
The practical benefits of the hyper-accuracy properties of the discontinuous Galerkin method are examined. In particular, we demonstrate that some flow attributes exhibit super-convergence even in the absence of any post-procesing technique. Theoretical analysis suggest that flow features that are dominated by global propogation speeds and decay or growth rates should be super-convergent. Several discrete forms of the discontinuous Galerkin method are applied to the simulation of unsteady viscous flow over a twodimensional cylinder. Convergence of the period of the naturally occurring oscillation is examined and shown to converge at $2 p+1$, where $p$ is the polynomial degree of the discontinuous Galerkin basis. Comparisons are made between the different discretizations and with theoretical analysis.
\end{abstract}

\section{Introduction}

The discontinuous Galerkin (DG) method provides a rigorous and robust means of formulating high-order methods for non-smooth unstructured grids. Such a method can be used to perform highly accurate simulations about complex geometries using grids that are readily generated. However, all high-order methods are more expensive than conventional second-order methods, on a per degree of freedom basis, so it is important to maximize the benefit of their high-order properties. The DG method is a projection technique in which the solution in each individual element is represented as an expansion in a local basis set; generally polynomials of degree $\leq p$. Formal proofs of error convergence ${ }^{1-3}$ predict that the DG method with a basis of degree $p$ should produce solutions that converge at a rate of $p+\frac{1}{2}$ on general grids, and at $p+1$ on non-smooth Cartesian grids and in several other special cases. Numerical experiments usually produce a convergence rate of $p+1$ even on most non-smooth unstructured grids.

However, there is a significant collection of analyses and numerical evidence indicating that the DG method provides a super-convergent solution of order $2 p+1$ or higher. ${ }^{4-10}$ In particular, a spatial eigenvalue analysis by $\mathrm{Hu}$ and Atkins ${ }^{7,8}$ for scalar convection and acoustic propagation clearly shows that the amplitude and phase errors converge respectively at rates of $2 p+1$ and $2 p+2$. The analysis demonstrated the superconvergence property for a range of $p$, and it was conjectured that it held for all $p$. This conjecture was later proven in Ref. 9. In Ref. 10, a spatial Fourier analysis of several variations of DG applied to diffusion for $p=4$ predicted the convergence of the temporal eigenvalue to be $p=9$ or $p=12$ depending on the form of the discretization. It has been conjectured that perhaps different DG discretizations converged at rates of $2 p+1$ or $3 p$. However, it will be shown in this work that this is not the case.

Several post-processing methods have been developed and demonstrated for model problems. ${ }^{6,11-16}$ Generally, these methods formally construct a solution of degree $2 p$ from a simulation of degree $p$ by computing the convolution of the solution against a specialized kernel function. However, there are several drawbacks to these techniques that limit their usefulness. Although the kernel is local and formally compact, the kernel stencil size is proportional to $p$, and therefore it can be quite large. Yet, it has been shown that the stencil must be small relative to any wavelength of interest in order to improve that mode of the solution. ${ }^{16}$ These methods usually require special grids that are difficult to apply to realistic flow simulations about complex

*Senior Research Scientist, Senior Member 
geometries. Finally, it is shown in Ref. 16 that some numerical errors, such as those produced by approximate numerical boundary conditions, can introduce errors that the post-processing kernel cannot distinguish from the correct physical solution. Consequently, such numerical errors cannot be removed by any local or compact post-processing technique.

Given that most post-processing techniques are difficult to successfully apply to a realistic simulation, it is reasonable to question whether the super-convergence property of the DG method is of any real value. As will be shown, the answer is yes; super-convergence is of real value. This paper examines the practical benefits of the super-convergence properties of the DG method. In particular, we demonstrate that certain flow attributes exhibit super-convergence in the absence of any post-procesing technique. Several discrete forms of the DG method are applied to the simulation of unsteady viscous flow over a two-dimensional cylinder. Convergence of the period of the naturally occurring oscillation is examined and compared with theoretical predictions.

The first section describes the implementation of DG to the Navier-Stokes equations. The second section reviews the superconvergence properties of the DG method for advection and diffusion equations, and presents additional new analysis for diffusion equations. The third describes the numerical experiments performed and their results.

\section{Governing Equations and DG Formulation}

\section{A. Navier-Stokes Equations}

The non-dimensional compressible Navier-Stokes equations in conservation form are:

$$
\begin{aligned}
\frac{\partial \rho}{\partial t} & +\frac{\partial\left(\rho u_{j}\right)}{\partial x_{j}}=0, \\
\frac{\partial \rho e}{\partial t} & +\frac{\partial\left(h u_{j}-u_{i} \tau_{i, j}+q_{j}\right)}{\partial x_{j}}=0, \\
\frac{\partial \rho u_{i}}{\partial t} & +\frac{\partial\left(\rho u_{i} u_{j}+\delta_{i, j} p-\tau_{i, j}\right)}{\partial x_{j}}=0,
\end{aligned}
$$

where $\rho$ is the density, $e$ is the internal energy per unit mass, $u_{i}$ is the component of velocity in the cartesian coordinate direction $x_{i}$,

$$
\begin{gathered}
h=\rho e+p, \\
q_{i}=\frac{-\gamma}{\gamma-1} \operatorname{Pr} \operatorname{Re}_{r}^{-1} \frac{\partial T}{\partial x_{i}}, \\
\tau_{i, j}=\mu \operatorname{Re}_{r}^{-1}\left(\frac{\partial u_{i}}{\partial x_{j}}+\frac{\partial u_{j}}{\partial x_{j}}-\delta_{i, j} \frac{2}{3} \frac{\partial u_{k}}{\partial x_{k}}\right),
\end{gathered}
$$

$T=p / \rho=(\gamma-1)\left(e-u_{k} u_{k} / 2\right), \operatorname{Pr}$ is the Prandtl number, and $R e_{r}$ is the Reynolds number based on reference state of the non-dimensionalization. The length and the thermodynamic variables have been nondimensionalized with respect to a general reference state: $x_{i}=\hat{x}_{i} / \hat{L}_{r}, \rho=\hat{\rho} / \hat{\rho}_{r}, p=\hat{p} / \hat{p}_{r}, T=\hat{T} / \hat{T}_{r}$, and $\mu=\hat{\mu} / \hat{\mu}_{r}$ where ${ }^{\wedge}$ denotes dimemsional quantities, and the subscript $r$ denotes the reference state. It follows that $u=\hat{u} / \hat{u}_{r}, t=\hat{t} /\left(\hat{L}_{r} / \hat{u}_{r}\right), e=\hat{e}_{r} / \hat{u}_{r}^{2}$, and $R e_{r} \equiv \hat{\rho}_{r} \hat{u}_{r} \hat{L}_{r} / \hat{\mu}_{r}$ where $\hat{u}_{r}=\sqrt{\hat{p}_{r} / \hat{\rho}_{r}}$.

DG is applied to each of the equations 1-3 in essentially the same manner; however, the inviscid and viscous terms are treated differently. To facilitate the following discussion, each equation is cast in the general form:

$$
\frac{\partial \tilde{U}}{\partial t}+\nabla \cdot\left(\tilde{\mathbf{F}}_{i}-\tilde{\mathbf{F}}_{v}\right)=0
$$

where $\tilde{U}$ denotes either $\rho, \rho e$, or $\rho u_{i}$, and $\tilde{\mathbf{F}}_{i}$ and $\tilde{\mathbf{F}}_{v}$ denote corresponding the inviscid and viscous contributions to the flux. Equation (4) is transformed to a local computational coordinate system for each discrete element. Let $(\xi, \eta, \zeta)$ denote the local coordinate system with Jacobian $J \equiv \partial\left(x_{1}, x_{2}, x_{3}\right) / \partial(\xi, \eta, \zeta)$. In the local coordinates, equation (4) has the same form:

$$
\frac{\partial U}{\partial t}+\nabla \cdot\left(\mathbf{F}_{i}-\mathbf{F}_{v}\right)=0
$$

but with $U=\tilde{U}|J|, F_{i}=J^{-1} \tilde{\mathbf{F}}_{i}|J|$, and $F_{v}=J^{-1} \tilde{\mathbf{F}}_{v}|J|$. 


\section{B. DG Discretization}

The computational domain is subdivided into non-overlapping elements that cover the domain. The DG discretization is formulated locally in each element in a similar manner. The solution within each element is approximated as an expansion in a local basis set $\left\{b_{k}\right\}$, usually polynomials of degree $\leq p$,

$$
U=\sum_{k=0}^{N} b_{k} u_{k}
$$

where $N$ denotes the number of terms in the basis set. The current work will uses two-dimensional monomials of the form $\xi^{i} \eta^{j}$ for all $(i, j)$ pairs satisfying $0 \leq i+j \leq p$. Following the quadrature-free formulation, ${ }^{17}$ the fluxes are expanded in a similar manner,

$$
\mathbf{F}_{i}=\sum_{k=0}^{M} b_{k} \mathbf{f}_{i, k} \quad \text { and } \quad \mathbf{F}_{v}=\sum_{k=0}^{M} b_{k} \mathbf{f}_{v, k}
$$

however, the degree of the flux expansions are allowed to be higher than that of the solution. The number of unknowns in each element is the number of physical variables times the size of the basis set, $N$. An equal number of equations governing these unknowns is derived by multiplying the governing equations by each member of the basis set, and integrating over the element. The integrals of the flux terms are integrated by parts to obtain the following weak form:

$$
\int_{\Omega} b_{k} \frac{\partial U}{\partial t} d \Omega-\int_{\Omega} \nabla b_{k}\left(\mathbf{F}_{i}-\mathbf{F}_{v}\right) d \Omega+\int_{\partial \Omega} b_{k}\left(\mathbf{F}_{i}-\mathbf{F}_{v}\right) \cdot \mathbf{n} d s=0 \quad \forall k: 0 \leq k \leq N,
$$

where $\Omega$ denotes the element, $\partial \Omega$ denotes the element boundary (or edge) and $\mathbf{n}$ denotes the outward unit normal. Because neighboring elements have independent local approximations for the solution and fluxes, the flux terms in the edge integrals are multi-valued. These fluxes are replaced with numerical fluxes that are functions of both the local and neighboring solutions.

$$
\left.\mathbf{F}_{i} \cdot \mathbf{n}\right|_{\partial \Omega} \equiv \widehat{F}_{i}=\sum_{k=0}^{N} b_{k} \widehat{f}_{i, k} \quad \text { and }\left.\quad \mathbf{F}_{v} \cdot \mathbf{n}\right|_{\partial \Omega} \equiv \widehat{F}_{v}=\sum_{k=0}^{N} b_{k} \widehat{f}_{v, k} .
$$

Because all of the fluxes are represented as expansions in the basis set, the integrations can be performed directly to produce a matrix equation of the form:

$$
\mathbf{M}\left[u_{k}\right]+\mathbf{V} \cdot\left[\mathbf{f}_{i, k}-\mathbf{f}_{v, k}\right]+\sum_{\text {edges }} \mathbf{B}_{e}\left[\widehat{\mathbf{f}}_{i, k}-\widehat{\mathbf{f}}_{v, k}\right]=0
$$

where $\mathbf{M}, \mathbf{V}$ and $\mathbf{B}$ are derived in Ref. 17.

Gradients of the solution required for the viscous terms are evaluated using the DG methodology in a similar manner. Let

$$
\boldsymbol{\sigma}=\sum_{k=0}^{N} b_{k} \boldsymbol{\sigma}_{k}=\nabla w
$$

where $w$ denotes either $u_{i}$ or $T$. It immedately follows that

$$
\int_{\Omega} b_{k} \boldsymbol{\sigma} d \Omega-\int_{\Omega} \nabla b_{k} w d \Omega+\int_{\partial \Omega} b_{k} w \mathbf{n} d s=0 .
$$

As before, the multi-valued edge flux is replaced by a numerical flux, $\left.w \mathbf{n}\right|_{\partial \Omega}=\widehat{w} \mathbf{n}$.

The inviscid edge flux is modeled by an approximate Riemann flux. The current work uses a Lax-Fredricks flux of the form:

$$
\widehat{F}_{i}=\left\{\mathbf{F}_{i}\right\} \cdot \mathbf{n}-\lambda \llbracket U \rrbracket / 2
$$

where $\{x\}$ denotes the average of $x, \llbracket x \rrbracket$ denotes the jump in $x$ (neighboring value minus local value), and $\lambda$ is the maximum absolute eigenvalue of the Jacobian of the system of inviscid fluxes. Quantities not explicitly inside an average or jump operator $(\{x\}$ or $\llbracket x \rrbracket)$ are evaluated using data from the local element. Note: 
because the outward normal, $\mathbf{n}$, is of the opposite sign in neighboring elements on a common edge, we have the identity: $\left\{\mathbf{F}_{i}\right\} \cdot \mathbf{n}=-\llbracket \mathbf{F}_{i} \cdot \mathbf{n} \rrbracket$.

Three variations of DG for the discretization of the Navier-Stokes equations are evaluated in this work. They differ only in the treatment of the numerical viscous fluxes as defined in Table 1 . The interiorpenalty formulation, ${ }^{20}$ denoted DG-IP, and the closely related method of Bassi ${ }^{21}$ that uses the "lifting" operator to evaluate $\alpha$, are the most commonly used forms of DG for Navier-Stokes simulations. The DG-IP discretization is compact, and it is perhaps the easiest in which to formulate exact Jacobians for use in implicit solvers and adjoint methods. The second and third forms are variations of the local-discontinuous Galerkin (LDG) method of Cockburn and Shu. ${ }^{18}$ The second method, obtained by setting $\beta=0$ (in Table 1), uses a symmetric edge flux in both the gradient and physical flux terms, and also adds a penalty term to the physical flux. This method will be denoted LDG-C (for LDG-central). The LDG-C spatial discretization is also easy to implement, but it results in a non-compact stencil which complicates the implementation of both implicit solvers and adjoint methods. The final method uses "one-sided" operators for the gradient and physical flux terms that alternate direction between the two. ${ }^{18,19}$ Hence, it is denoted as LDG-OS. It also results in a compact discretization; however, its implementation is slightly more complex than that of the DG-IP method. It is obtained by setting $\beta= \pm 1 / 2$ and $\alpha=0$. The sign of $\beta$ is determined by imposing a global, but otherwise nearly arbitrary, ordering on the elements, and then choosing the sign according to whether the local element is "above" or "below" its neighbor.

\section{Non-linear Flux Terms}

The quadrature-free form of DG requires that all fluxes be expanded in the basis set. This process is trivial and exact when the equations are linear, but must be approximated for most non-linear cases. Previously, ${ }^{22,23}$ it was noted that the inviscid fluxes of the Euler equations consist of ratios of polynomials such as

$$
f=\frac{\left(\rho u_{i}\right)\left(\rho u_{j}\right)}{\rho} \text { or } f=\frac{\left(\rho u_{i}\right)(\rho e)}{\rho} .
$$

Polynomial approximations to the flux can be forumulated by multiplying by $\rho$, and projecting back into the polynomial space. For example:

$$
\int b_{k} \rho f=\int b_{k}\left(\rho u_{i}\right)\left(\rho u_{j}\right)
$$

Considering only design order arguments, the polynomial products can be truncated to degree $p$, and the projection can be limited to $k \leq N$. However, in Ref. 23 it was shown that extending the degree of the flux improved robustness in cases of shock capturing. In the current work, polynomial products are truncated to $p+1$, and the projection is over that same set of functions. The operation is efficiently performed by noting that the left-hand side is the same for all terms,

$$
\text { LHS }=\left[\int b_{i} b_{j} \rho\right]
$$

allowing the $L U$-decomposition of the matrix to be re-used many times.

The $u_{i}$ and $T$ terms, needed to compute the viscous gradients, are obtained in a similar manner by

\begin{tabular}{|c|c|c|}
\hline Scheme & $\widehat{w}$ & $\widehat{f_{v}}$ \\
\hline $\begin{array}{l}\text { LDG-C and LDG-OS }{ }^{18,19} \\
\text { DG-IP }^{20}\end{array}$ & $\begin{array}{c}\{w\}-\beta \cdot \llbracket w \rrbracket \\
\{w\}\end{array}$ & $\begin{array}{l}\left\{f_{v}\right\}+\beta \llbracket f_{v} \rrbracket-\alpha \llbracket w \rrbracket \\
\quad\{\nabla w\}-\alpha(\llbracket w \rrbracket)\end{array}$ \\
\hline
\end{tabular}
solving:

$$
\int b_{k} \rho f=R, \text { where } R=\int b_{k}\left(\rho u_{i}\right) \text { or } \int b_{k} P .
$$

Table 1. Numerical viscous fluxes. 


\section{Super-convergence properties of DG}

For a DG solution that is an expansion in a finite polynomial basis, say degree $=p$, any direct discrete comparison with an exact solution will clearly result in a measured error that converges no faster than $p+1$. It would seem that some post-processing would be necessary in order to obtain any real benefit. However, previous spatial eigenvalue/eigenfunction analysis of acoustic propagation ${ }^{7,8}$ have definitively demonstrated that the eigenvalues of the DG solution are super-convergent. In its simplest form, this analysis assumes that the discrete eigensolution is of the form

$$
\mathbf{u}_{h}^{n}(\xi, t)=e^{-i \omega t} \lambda^{n} u(\xi)
$$

where $\lambda=e^{i K_{h}}$ and $u(\xi)$ is a polynomial function of degree $p$ within a single element at $n=0$. Substitution into a model wave equation, with wave speed $a$, produces a dispersion relation that governs the eigensolution. For the compact DG discretization, this equation has the form:

$$
(1-\gamma)[G(i K) \lambda-H(i K)]+(-1)^{p+1}(1+\gamma)\left[G(-i K) \frac{1}{\lambda}-H(-i K)\right]=0
$$

where $K=\frac{\omega h}{a}$, and $H$ and $G$ are polynomial functions of the normalized frequency, $K$, and $\gamma$ arises out of a generalized representation of several flux splitting formulations, as described in Ref. 7. A conventional Fourier analysis sets the wave number, $K_{h}$, and solves for the resulting temporal behavior. However, this results in an eigenvalue problem of size $p+1$ that must be solved numerically, and that produces multiple solutions that require some interpretation. As noted in Ref. 7, equation (7) gives a simple quadratic relation for $\lambda$ that can be solved analytically when $\omega$ is given. One root corresponds to a non-physical mode that has been shown to be damped such that it does not interfere with the properties of the asymptotic physical solution. It was also shown that the ratio of polynomials, $H(i K) / G(i K)$, equals the exact Pade approximation of $e^{i K}$ to order $2 p+2$; and consequently, the physically significant root is of order:

$$
\lambda=e^{i K}+O\left((\omega h)^{2 p+2} .\right.
$$

Thus, the dispersion and dissipation errors converge as $\operatorname{Re}\left(i K_{h}\right) \sim K+O\left((\omega h)^{2 p+3}\right)$ and $\operatorname{Im}\left(K_{h}\right) \sim$ $O\left((\omega h)^{2 p+2}\right)$, respectively. Note that because the analysis is for a single time step (or cell translation), the convergence rate of the error after integrating to a fixed time is one order lower.

In Ref. 10, a Fourier analysis of DG applied to diffusion indicated that all three discretizations described in the previous section have a super-convergence property. In that work, the analysis was performed for $p=4$ only, and it was found that the DG-IP discretization converged at a rate of $p=9$, while the LDG-C and LDG-OS discretizations converged at a rate of $p=12$. It has been conjectured that this implied general rates of $2 p+1$ for DG-IP and $3 p$ for LDG-C and LDG-OS.

Here the earlier analysis is extended to include $p=1,2$, and 3 in order to determine the true convergence properties. The procedure is similar to the analysis described above (ref. 7 ) except that here we choose $K_{h}$ and solve governing equations for $K$. After including the diffusion terms, the governing equations define a matrix eigenvalue problem of size $p+1$ that must be solved numerically for the eigenvalues, $K_{k}$. Each of the multiple solutions for $K_{k}$ obtained at a given $K_{h}$ correspond to spatial wave number that is shifted by some multiple of $\pi$ (e.g. $K_{h}+k \pi$ ). Rigorous association of $K_{k}$ with the appropriate wave number would require examining the wavelength of the eigenfunction. However, for the current purpose of inferring order properties, it is generally sufficient to simply sort the solutions from smallest to largest, $\left(K_{i}<K_{j}\right.$ if $i<j$ ) and associate each solution with a wave number as $\left[K_{k}, K_{h}+k \pi\right]$. Figures 1 a-c show the result of this analysis for each of the three discretizations, and for $p=1-4$. The dashed line provides reference convergence rates. The curves are labeled $p=a(b)$ where $a$ is the degree of the basis set, and $b$ is the convergence rate of the reference line. From these results it is clear that DG-IP and LDG-C converges at $2 p+2$, and LDG-OS converges at $2 p+4$. As before, these rates are for a single time step, and the convergence rates at a fixed time would be one order lower (assuming $\delta t \propto h$ ).

Both analyses predict a super-convergent relationship between the temporal and spatial eigenmodes (i.e. between $e^{-i \omega t}$ and $\lambda$ ), but say nothing about the function $u(\xi)$. In fact, $u(\xi)$ is simply a polynomial function of degree $p$ that is completely determined by initial and boundary conditions; and than evolves in time and space as governed by $e^{-i \omega t} \lambda^{n}$. Post-processing techniques are generally local and compact, and can be used to construct a higher-order local solution. However, because post-processors are local and compact, 


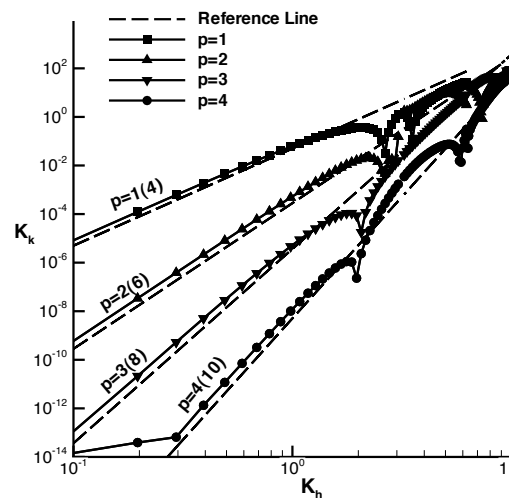

(a) DG-IP

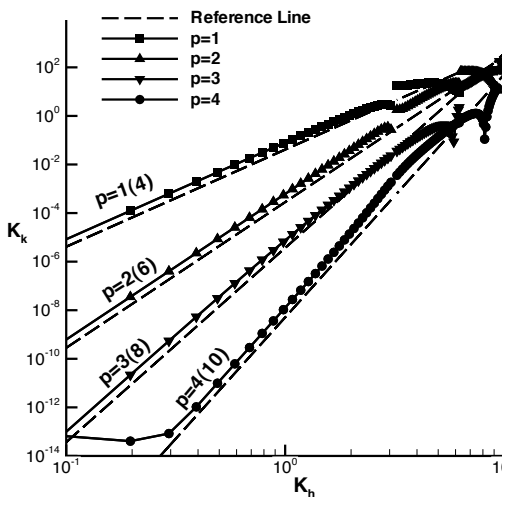

(b) LDG-C

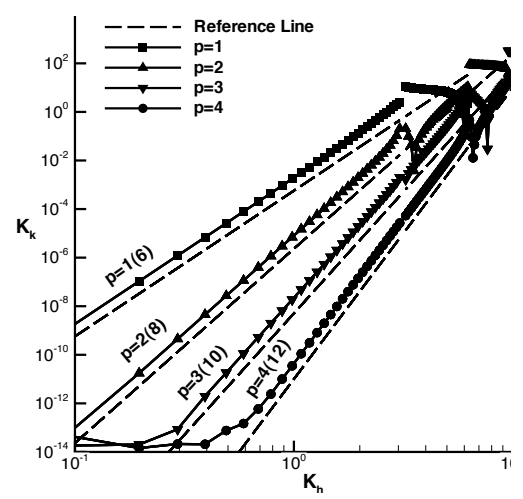

(c) LDG-OS

Figure 1. Convergence rate predicted by Fourier Analysis

they cannot correct the global dispersion and dissipation errors. The global super-convergence properties are inherent to the DG discretization method, and are a prerequisite for any successful post-processing technique. In essence, local post-processing techniques can only improve $u(\xi)$ by "smoothing" high-order noise in the local solution.

This analysis has several implications for realistic simulations. First, it implies that the accumulation of error remains small for features that evolve for long periods of time or space, perhaps smaller than the initial and boundary error. Thus for the scatter of acoustic waves off of a complex geometry, one could expect super-convergence of the wave propagation and the resulting interference pattern. Also, the growth of transitional and weak instability modes that evolve for long times and distances may be super-convergent.

Second, this analysis suggests that naturally occurring phenomena that depend weakly on initial and boundary conditions may be super-convergent. For example, the profile of a boundary layer on a flat plate may be super-convergent; especially since the shape of the geometry and the constant pressure outer condition can be represented exactly by even the lowest order DG basis. Viscous layers over more complex, yet sufficiently smooth, geometries may also be super-convergent. Finally, naturally occurring unsteadiness such as that in bluff body and cavity flows, as well as transitional instabilities, may be super-convergent.

\section{Test Case}

\section{A. Model Problem}

Viscous flow over a two-dimensional cylinder is chosen as a test case because the flow produces a well behaved natural unsteadiness at a dominant frequency. In addition, the geometry is simple and analytic, but nontrival (e.g. it cannot be represented exactly by the polynomial basis.) Previous investigations concerning the super-convergence of acoustic scatter ${ }^{10}$ suggest this boundary shape is sufficiently smooth so as not to obscure any super-convergence behavior that may exist.

Figures 2a-c illustrate typical grids showing the full domain on the coarsest grid, and the coarsest and finest grids near the cylinder. Simulations were performed on a sequence of four grids with the number of elements around the half cylinder, $N x$, equal to $5,10,20$, and 40 , for $p \leq 3$; and with $N x=9,12,18$, and 27 for $p=4$. Although the grids shown have straight edges, element edges lying on the cylinder are curved and defined by projections onto a polynomial space. Points 1,2 , and 3 , shown in figure 2(b), are the locations at which the flow properties were sampled.

The freestream Mach number, $M_{\infty}$, is 0.2 and the Reynolds number, $R e$, is 200 , based on the cylinder diameter, $d$, and freestream velocity. Taking the reference state as freestream, the reference Reynolds number is given by,

$$
R e_{r} \equiv \frac{\hat{\rho}_{r} \hat{u}_{r} \hat{L}}{\hat{\mu}_{r}}=\left(\frac{\hat{\rho}_{\infty} \hat{u}_{\infty} \hat{d}}{\hat{\mu}_{\infty}}\right)\left(\frac{\hat{u}_{r}}{\hat{u}_{\infty}}\right)\left(\frac{\hat{L}}{\hat{d}}\right)
$$




$$
=\operatorname{Re}\left(\frac{\hat{L}}{\hat{d}}\right) /\left(\sqrt{\gamma} M_{\infty}\right) .
$$

The non-dimensional viscosity, $\mu$, is held constant at 1 .

The simulations are started impulsively from a modified freestream. Near the cylinder, the flow velocity is reduced linearly in the radial direction such that the initial solution satisfies the no-slip condition. Additionally, a compact, low amplitude vortical component is added to the initial solution in the neighborhood of the cylinder to excite the natural oscillation, and reduce the cost of the simulation.

\section{B. Error and Convergence Measurement Techniques}

The truncation error of any simulation is usually assumed to behave as

$$
U_{k}-U_{\text {exact }}=\sum_{n=r}^{\infty} \alpha_{n}\left(h_{k} / h_{0}\right)^{n}
$$

where $k$ identifies a grid within a consistent family of grids. For sufficiently small $h_{k} / h_{0}$, the asymptotic rate of convergence is given by

$$
n \approx r_{k}=\log \left[\frac{U_{k}-U_{\text {exact }}}{U_{k-1}-U_{\text {exact }}}\right] / \log \left(h_{k} / h_{k-1}\right) .
$$

Of course in most realistic cases, $U_{\text {exact }}$ is not known. It is common to use a very fine grid solution as a surrogate for the exact solution; however, this can be prohibitively expensive. A practical alternative is simply to note that the expression

$$
U_{k}-U_{k+1}=\sum_{n=r}^{\infty} \alpha_{n}\left(1-\left(h_{k+1} / h_{k}\right)^{n}\right)\left(h_{k} / h_{0}\right)^{n}
$$

converges at the same asymptotic rate as true error given in equation (8). Thus, another approximation for the asymptotic rate is given by

$$
r_{k}=\log \left[\frac{U_{k}-U_{k+1}}{U_{k-1}-U_{k}}\right] / \log \left(h_{k} / h_{k-1}\right) .
$$

The common practice of simply "fitting" the asymptotic error equation to the solutions on a sequence of three grids results in the same formula. This approximation for the convergence rate is accurate only if all three grids used in the evaluation of equation (11) are within the asymptotic regime. Note that nonmonotonic convergence, $\left(U_{k}-U_{k+1}\right) /\left(U_{k-1}-U_{k}\right)<0$, is a clear indicator that the coarser grids are not in the asymptotic regime.

A third approach, described in Ref. 24 and 25, uses a sequence of 4 grids to relax this requirement by modeling the error as

$$
\Delta_{k} \equiv U_{k}-U_{k+1}=\alpha_{r}\left(h_{k} / h_{0}\right)^{r}+\alpha_{r+1}\left(h_{k} / h_{0}\right)^{r+1} .
$$

The convergence rate is approximated by

$$
r_{k}=\log _{2}\left[\frac{3 \Delta_{k+1}}{4 \Delta_{k+2}} \pm \sqrt{\left(\frac{3 \Delta_{k+1}}{4 \Delta_{k+2}}\right)^{2}-\frac{\Delta_{k}}{2 \Delta_{k+2}}}\right], \quad \text { where } \quad h_{k-1} / h_{k}=2 \forall k .
$$

Here, this formula is generalized to arbitrary mesh ratios, $\sigma \equiv h_{k-1} / h_{k}$ as:

$$
r_{k}=\log _{\sigma}\left[\frac{\Gamma \Delta_{k+1}}{\Delta_{k+2}} \pm \sqrt{\left(\frac{\Gamma \Delta_{k+1}}{\Delta_{k+2}}\right)^{2}-\frac{\Delta_{k}}{\sigma \Delta_{k+2}}}\right],
$$

where $\Gamma=(\sigma+1) /(2 \sigma)$. If all 4 grids are in or near the asymptotic regime, then $\Delta_{k+1} / \Delta_{k+2}>0$, and "+" is the appropriate sign before the radical. References 24 and 25 give a quantitate means of checking the validity of equation (14) by computing the ratio of the high- and low-order terms in equation (12).

$$
\frac{\alpha_{r} h_{k}}{\alpha_{r+1}}=\frac{4\left(1-2^{p}\right)}{\left(1-2^{p+1}\right)} \frac{\left(\Delta_{k}-2^{p} \Delta_{k+1}\right)}{\left(2^{p+1} \Delta_{k+1}-\Delta_{k}\right)} .
$$




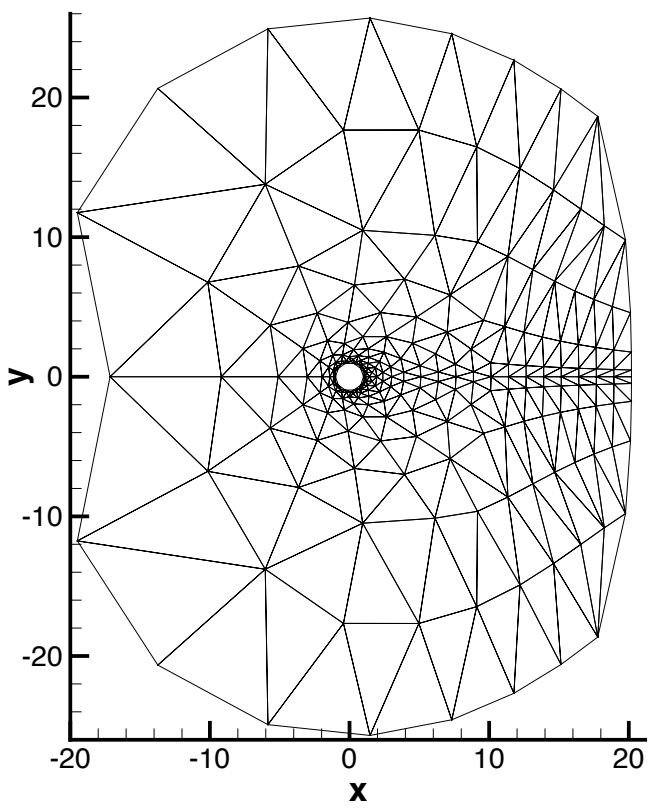

(a) coarsest grid, full domain

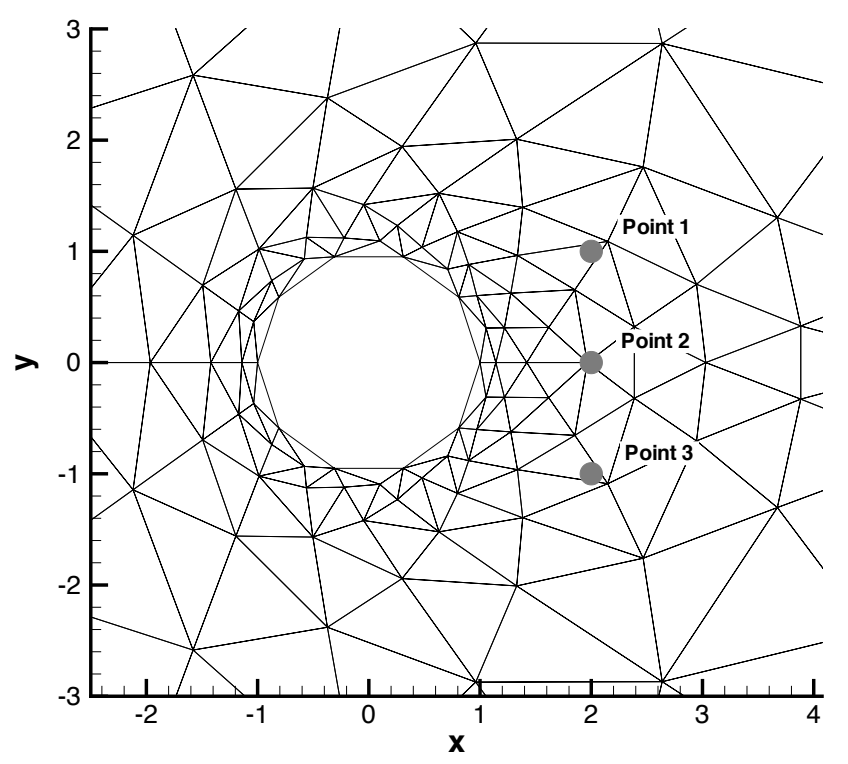

(b) coarsest grid near cylinder

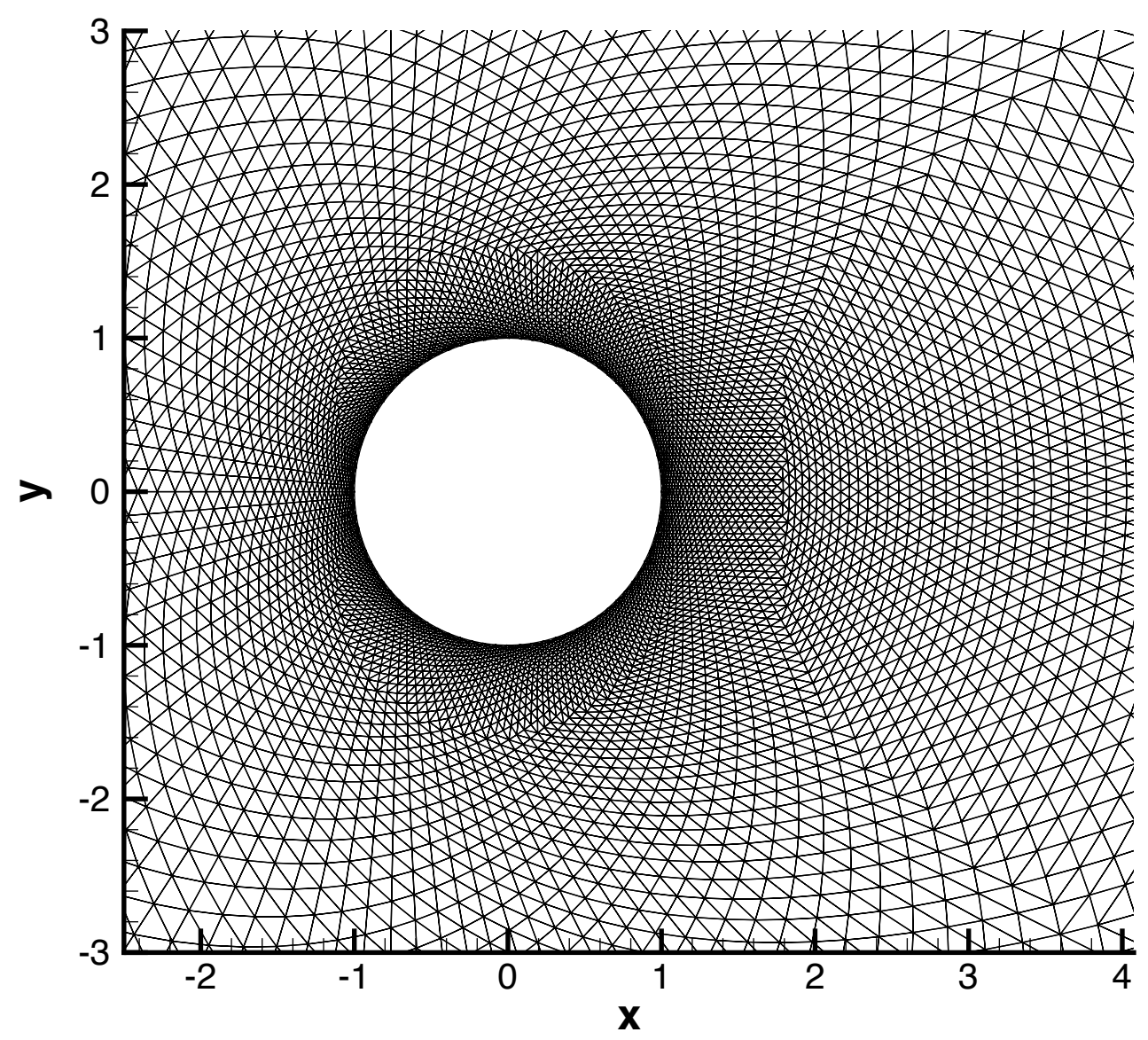

(c) finest grid near cylinder

Figure 2. Computational doman with typical coarsest and finest grids 
If this term is small $(<O(1))$, then it is likely that all 4 grids are within or near the asymptotic regime. However, if it is much greater than 1, then it is likely that higher-order terms neglected by equation (12) are contributing to the error on the coarser grids, and that equation (14) is unreliable. The formulas given in equations 11 and 14 will be referred to in the remaining discussion as 3-grid and 4-grid approximations, respectively.

\section{Numerical Test}

The three variations of DG described above are applied to simulate unsteady viscous flow over a twodimensional cylinder. For each discretization method, the solution is approximated by a polynomial basis of degree $p=1,2,3$, or 4 , which should result in a design order of accuracy of $p+1=2,3,4$, or 5 , respectively. Simulations on a sequence of 4 grids were performed for each discretization method and for each basis degree.

All flow solution variables were monitored at the three sample points shown in figure 2(b). The value of $\rho v$ at point 3 will be used to illustrate typical results in the remaining discussion. Figure 3 compares the evolution of the $p=1$ solution on the coarsest grid with the $p=3$ solution on the finest grid. Both solutions experience a brief transient, but settle to a quasi-periodic solution after 6-8 cycles. There is a significant difference in the period between the two solutions: approximately $30 \%$.

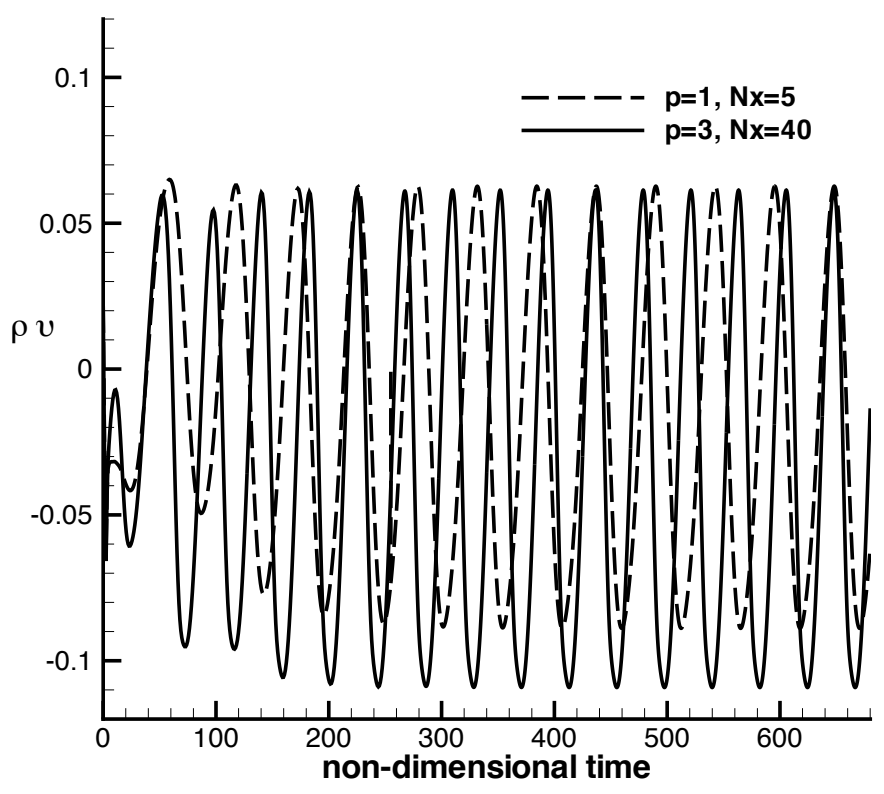

Figure 3. Evolution of $\rho v$ component at point 3.

The period, $\lambda$, is computed as the time interval between events when the solution crosses a specified value. The specified value varies with each sample point location and flow variable. Even though the time step is extremely small due to the explicit time marching method used, a high order interpolation is used to determine the precise moment of this crossing event between time steps. Before comparisons can be made between solutions on different grids, it is necessary confirm that transients in the solutions are small relative to the differences measured between grids. Figure 4 provides a measure of the decay of non-periodic transients by showing the change in the period between successive periods for all grids, basis degrees, and discretizations. Results for the different discretizations are shown without distinction, and generally cannot be distinguished from one another except at isolated points. Generally speaking, transients in all grids, degrees, and discretizations decay at similar rates, but the more accurate solutions are less monotone in 
their convergence.

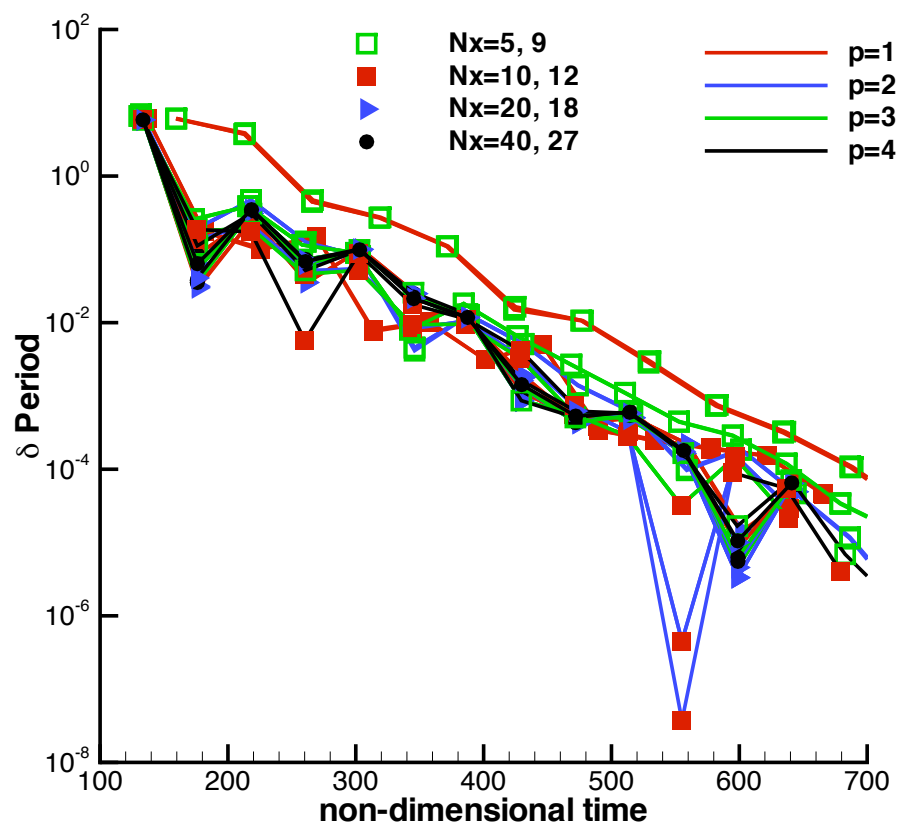

Figure 4. Decay of non-periodic transients. Results from different discretizations are shown without distinction.

The change in the period with mesh refinement, $\lambda_{k}-\lambda_{k-1}$, is shown in figure 5 for all grids, degrees, and discretizations. Again, the different discretizations are shown without distinction. The higher-order cases require $9-11$ periods before this metric begins to reach stable values. This is expected because the flow transients, shown in figure 4, must decay to levels well below the change in period with mesh refinement. After the initial transients decay, the results from the different discretizations overlay one another. Later in this discussion, subsets of this collection illustrating typical results for single discretization will be isolated and examined separately.

Grid convergence of $\lambda_{k}-\lambda_{k-1}$ is shown in figures 5. Because all three discretizations give similar results for $p \leq 3$, only for LDG-OS computations were performed at $p=4$. Recall that $\lambda_{k}-\lambda_{k-1}$ converges asymptotically at the same rate as the true error. Also note that if the error on a sequence of grids is in the asymptotic regime, then the curves will be uniformly spaced when viewed on a log plot. However, only the $p=1$ cases (figure 5a) display this behavior. This indicates that all four grids are within the asymptotic regime for all cases with degree $p=1$. Also, note that for $p=3$ (figure $5 \mathrm{c}$ ) the change in the period is small for the coarse grid cases $(N x=10)$. This does not mean that the error in the period is small; only that the change in the period between the two coarsest grids is small. This generally indicates that the convergence is not monotone, and that the coarsest grids are well outside of the asymptotic regime for $p=3$. Directly examining the data for $p=2$ (figure $5 \mathrm{~b}$ ) reveals that the convergence is highly oscillatory. The sign of the change in the period flips between every mesh refinement (positive for $N x=10$ and 40, negative for $N x=20$ ). This suggest that all of the grids may be well outside of the asymptotic regime, and that more than two error modes are contributing to the error on most, if not all, grids. Therefore, neither the 3 -grid or the 4-grid approximation for the convergence rate can be applied to the $p=2$ case.

Convergence rates for LDG-OS, computed using both 3-grid and 4-grid approximations, are shown in figure 6 . Cases with $p=1,3$, and 4 super-converge at a rate approaching $2 p+1$ that is steady beyond the 11th cycle $(t>400)$. The 3 -grid approximation tends to be lower than the 4-grid approximation by an amount that increases with $p$. This is probably because the higher order discretizations require finer grids to be firmly in the asymptotic regime, and the 3-grid approximation is more strongly influenced by neglected 


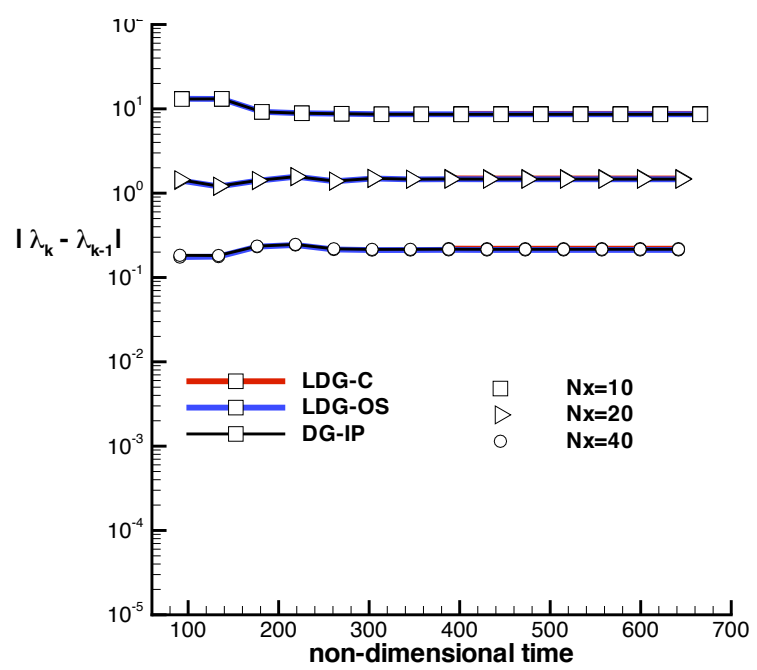

(a) $p=1$

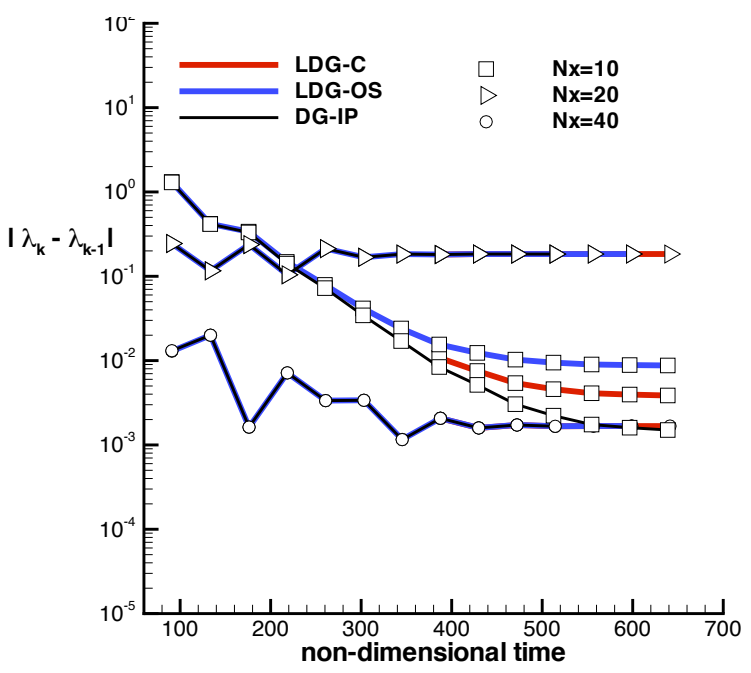

(c) $p=3$

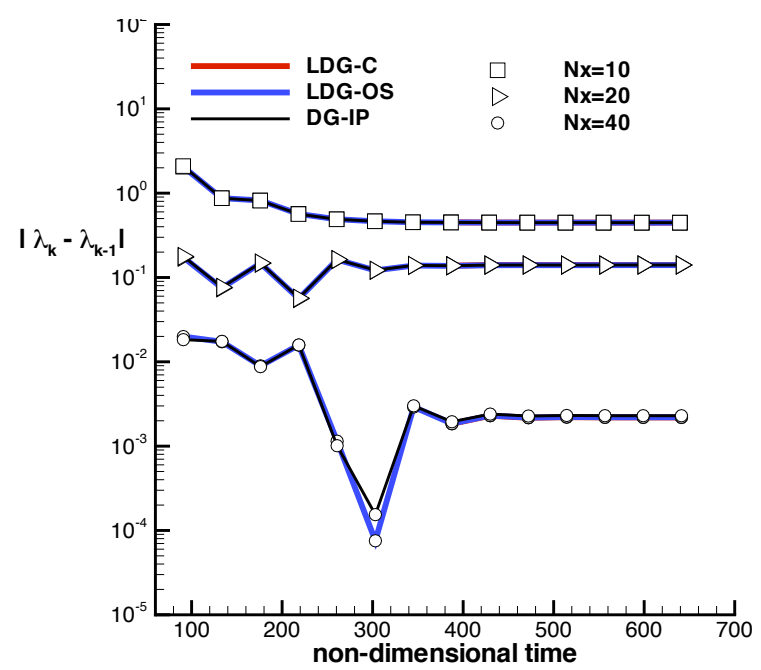

(b) $p=2$

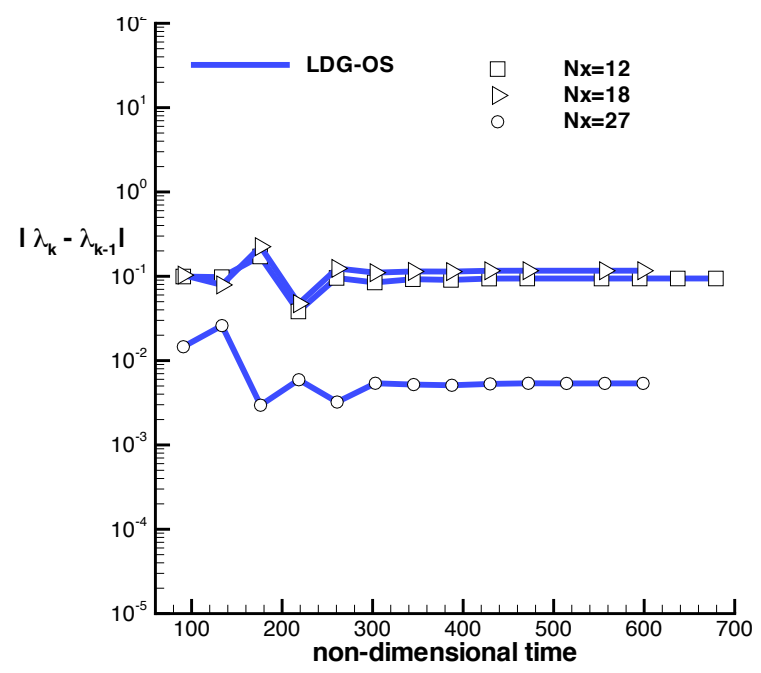

(d) $p=4$

Figure 5. Change in period with mesh refinement. 
terms. Because the $p=1$ case is converging uniformly on all 4 grids, the 3-grid approximation can be applied to both the three coarser grids, and the three finer grids. The two computations give the similar results and both results are shown without distinction (the solid red squares.) Again, neither approximation for the rate of convergence is directly applicable to the $p=2$ case; however, the absolute levels of the change in the period are similar to those of the $p=3$ case.

Although there are theoretical reasons to expect that LDG-OS might exhibit a super-convergent rate of $2 p+3$, it was not observed in these numerical experiments. This may be because the inviscid terms, which are treated the same in all cases, are the limiting contribution to the error. It is known that the Strouhal number is essentially constant over a range of Reynolds and Mach numbers, which means the shedding frequency is essentially proportional to the flow velocity. Although the viscous layer plays a vital roll in vortex formation, it is likely that the frequency of shedding is strongly influenced by the rate at which vortices advect downstream, and acoustic feedback travels upstream to the cylinder.

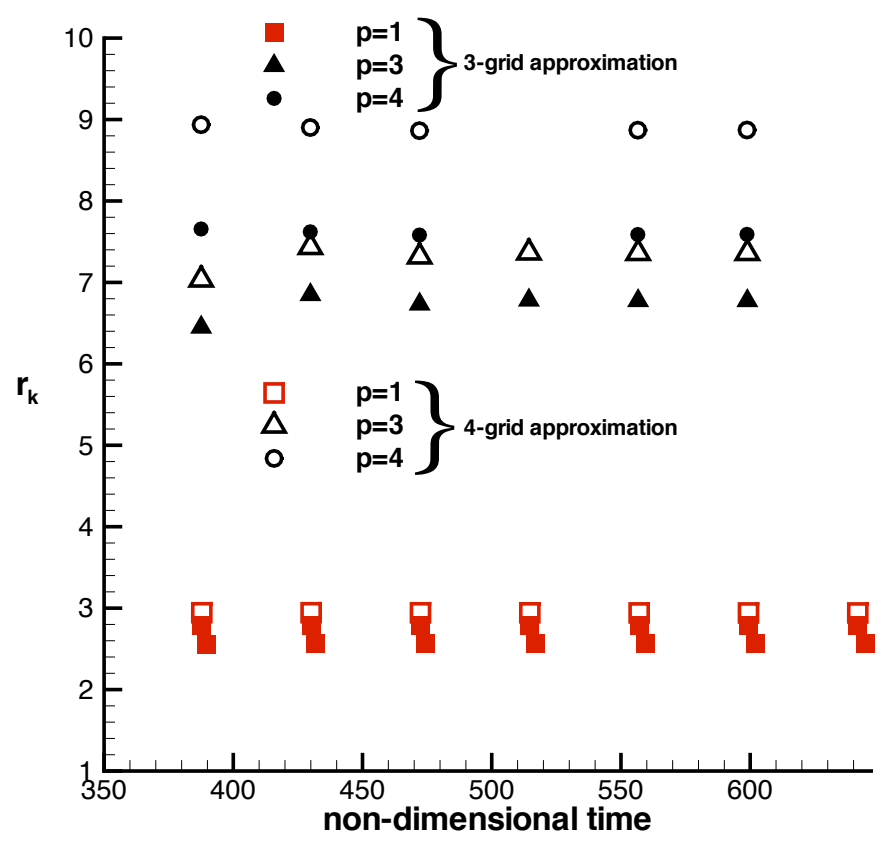

Figure 6. Convergence rate of LDG-C predicted by 3-grid and 4-grid approximations.

\section{Conclusions}

The several discrete forms of the discontinuous Galerkin method have been applied to an unsteady viscous flow to evaluate the convergence rates of naturally occurring oscillations. Numerical tests confirm that the super-convergence predicted by analysis can be realized for all three discretizations. The period computed by all discrete forms of discontinuous Galerkin for $p=1,3$ and $p=4$ converge at a rate approaching $2 p+1$. The change in period with mesh refinement oscillates in sign for the $p=2$ case, and formulas for the convergence rate do not directly apply. Although the change in period with mesh refinement for $p=2$ is similar to that of $p=3$, the error of all $p=2$ cases is probably much larger than that of $p=3$. These results clearly demonstrate that important flow features from simulations using the discontinuous Galerkin method are super-convergent without the application of any post-processing technique. 


\section{References}

${ }^{1}$ Johnson, C. and Pikäranta, J., "An analysis of the discontinuous Galerkin method for a scalar hyperbolic equation," Math. Comp., Vol. 46, 1986, pp. 1-26.

${ }^{2}$ Richter, G., "An optimal-order error estimate for the discontinuous Galerkin method," Math. Comp., Vol. 50, 1988, pp. $75-88$.

${ }^{3}$ LeSaint, P. and Raviart, P., "On a finite element method for solving the neutron transport equation," Mathematical aspects of finite elements in partial differential equations, edited by C. d. Boor, Academic Press, 1974, pp. 89-145.

${ }^{4}$ Adjerid, S., Aiffa, M., and Flaherty, J., "High-order finite element methods for singularly-perturbed elliptic and parabolic problems," SIAM J. Appl. Math., Vol. 55, 1995, pp. 520-543.

${ }^{5}$ Lowerie, R., "Compact higher-order numerical methods for hyperbolic for hyperbolic conservation laws," PhD. thesis, University of Michigan, 1996.

${ }^{6}$ Cockburn, B., Luskin, M., Shu, C.-W., and Suli, E., "Post-processing of Galerkin methods for hyperbolic problems," Discontinuous Galerkin Methods: Theory, Computation and Applications, edited by B. C. G. Karniadakis and C.-W. Shu, Vol. 11 of Lecture Notes in Computational Science and Engineering, Springer, 1999, pp. 291-300, Proceedings of the International Symposium on Discontinuous Galerkin Methods, Newport, May 1999.

${ }^{7} \mathrm{Hu}, \mathrm{F} . \mathrm{Q}$. and Atkins, H. L., "Eigensolution analysis of the discontinuous Galerkin method. Part I: One space dimensions," J. Comput. Phys., Vol. 182, 2002, pp. 516-545.

${ }^{8} \mathrm{Hu}$, F. Q. and Atkins, H. L., "Two-dimensional Wave Analysis of the Discontinuous Galerkin Method with Non-Uniform Grid and Boundary Conditions," AIAA paper 2002-2514, 8th AIAA/CEAS Aeroacoustics Conference and Exhibit, Breckenridge, CO, June 2002.

${ }^{9}$ Ainsworth, M., "Dispersive and dissipative behaviour of high order discontinuous Galerkin finite element methods," $J$. of Comp. Physics, Vol. 198, No. 1, 2004, pp. 106-130.

${ }^{10}$ Helenbrook, B. T. and Atkins, H., "Application of p-Multigrid to Discontinuous Galerkin Formulations of the Poisson Equation," AIAA Journal, Vol. 44, No. 3, 2006.

${ }^{11}$ Cockburn, B., Luskin, M., Shu, C.-W., and Süli, E., "Enhanced accuracy by post-processing for finite element methods for hyperbolic equations," Mathematics of Computation, Vol. 73, 2003, pp. 557-606.

${ }^{12}$ Adjerid, S., Devine, K., Flaherty, J., and Krivodonova, L., "A posteriori error estimation for discontinuous Galerkin solutions of hyperbolic problems," Comput. Methods Appl. Mech. Engrg., Vol. 191, 2002, pp. 1097-1112.

${ }^{13}$ O.C., Z. and Zhu, J., "The super-convergence patch recovery and posteriori error estimates. Part 1: The recovery technique," Int. J. Numer. Methods Engrg., Vol. 163, 1998, pp. 159-170.

${ }^{14}$ Ryan, J., "Extension of a Post-Processing Technique for Discontinuous Galerkin Finite Element Methods for Hyperbolic Equations," PhD. thesis, Brown University, 2003.

${ }^{15}$ Ryan, J., Shu, C.-W., and Atkins, H., "Extension of a Post Processing Technique for the Discontinuous Galerkin Method for Hyperbolic Equations with Application to an Aeroacoustic Problem," SIAM Journal on Scientific Computing, Vol. 26, No. 3, 2005, pp. 821-843.

${ }^{16}$ Atkins, H. L., Ryan, J. R., and Shu, C.-W., "Application of an accuracy enhancing post-processing method to aeroacoustic solutions," AIAA Paper 2003-3115, May 2003, 9th AIAA/CEAS Aeroacoustics Conference and Exhibit, Hilton Head, SC.

${ }^{17}$ Atkins, H. L. and Shu, C. W., "Quadrature-Free Implementation of Discontinuous Galerkin Method for Hyperbolic Equations," AIAA Journal, Vol. 36, No. 5, 1998, pp. 775-782.

${ }^{18}$ Cockburn, B. and Shu, C.-W., "The Local Discontinuous Galerkin Method for Time-Dependent Convection-Diffusion Systems," SIAM Journal of Numerical Analysis, Vol. 35, No. 6, 1998, pp. 2440-2463.

${ }^{19}$ Atkins, H. L. and Shu, C.-W., "Analysis of the Discontinuous Galerkin Method Applied to the Diffusion Operator," AIAA Paper 99-3306, 1999, 14th AIAA Computational Fluid Dynamics Conference, Norfork, Virginia, June 28-30.

${ }^{20}$ Douglas, J, J. and Dupont, T., "Interior Penalty Procedures for Elliptic and Parabolic Galerkin Methods," Proceedings of the Second International Symposium on Computing Methods in Applied Sciences and Engineering, 2d. Versailles, 1975, edited by R. Glowinski and J. L. Lions, Vol. 58 of Lecture Notes in Physics, Springer-Verlag, 1976.

${ }^{21}$ Bassi, F., Rebay, S., Mriotti, G., Pedinotti, S., and Savini, M., "A high-order accurate discontinuous finite element method for inviscid and viscous turbomachinery flows," proceedings of the 2nd European Conference on Turbomachinery, Fluid Dynamics, and Thermodynamics, edited by R. Decuypere and G. Diebelius, Lecture Notes in Mathematics, Technologisch Instituut, Antwerpen, Belgium, October 1997, pp. 99-108.

${ }^{22}$ Atkins, H. L., "Quadrature-Free Implementation of Discontinuous Galerkin Method for Hyperbolic Equations," AIAA Paper 96-1683, May 1996, 2nd AIAA/CEAS Conference.

${ }^{23}$ Atkins, H. L., "Local Analysis of Shock Capturing Using Discontinuous Galerkin Methodology," AIAA Paper 97-2032, 1997, 13th AIAA Computational Fluid Dynamics Conference, Snowmass Village, Colorado, June 29-July 2.

${ }^{24}$ Salas, M. D. and Atkins, H. L., "On problems associated with grid convergence of functionals," Computers $\mathcal{E}$ Fluids, Vol. 38, 2009, pp. 1145-1454.

${ }^{25}$ Salas, M. D. and Atkins, H. L., "Problems associated with grid convergence of functionals," Fifth International Conference on Computational Fluid Dynamics, Seoul, Korea, July 7-11, 2008. 